\title{
Fission description: First steps towards a full resolution of the time-dependent Hill-Wheeler equation
}

\author{
Marc Verrière $^{1, \mathrm{a}}$, Noël Dubray ${ }^{1, \mathrm{~b}}$, Nicolas Schunck $^{2}$, David Regnier ${ }^{1}$, and Pierre Dossantos-Uzarralde ${ }^{1}$ \\ 1 CEA, DAM, DIF, 91297 Arpajon, France \\ 2 Nuclear and Chemical Science Division, Lawrence Livermore National Laboratory, Livermore, CA 94551, USA
}

\begin{abstract}
Dynamical description of low energy fission is, in our full microscopic approach, decomposed in two steps. In the first step we generate the Potential Energy Surface (PES) of the compound system we want to describe with the Hartree-Fock-Bogoliubov (HFB) method with a Gogny interaction. The second step uses the Time Dependent Generator Coordinate Method (TDGCM) with the Gaussian Overlap Approximation (GOA). The GOA holds in two assumptions: the overlap matrix between HFB states has a gaussian shape (with respect to the difference between coordinates of states in deformation space); and the expectation value of the collective hamiltonian between these states can be expanded up to order two, leading in this case to a Schrödinger-like equation.

In this work we replace TDGCM+GOA in the second step of our approach by an exact treatment of the TDGCM. The main equation of this method is the time-dependent Hill-Wheeler equation and involves two objects: the overlap matrix and the collective hamiltonian. We first calculate these matrices on a PES. Then, we build an "exact TDGCM" solver using a finite element method and a Crank-Nicolson scheme.

In this talk, we will present the time-dependent Hill-Wheeler equation and discretization schemes (in time and deformation space). The analytic calculation of overlap matrix and collective hamiltonian will be detailed. Finally, first results with an exact treatment of the TDGCM will be discussed.
\end{abstract}

\section{Introduction}

Several approaches have been using for the last eighty years for the description of the fission dynamics:

- the micro-macro ones [1,2], where the nucleus is represented by a classical system (a liquid drop for example) with microscopic corrections;

- the microscopic ones, for exemple TDHF [3-5] and the TDGCM [6-8] where an effective nucleon-nucleon interaction is postulated and the dynamics is obtained by solving the Schrödinger equation.

In our case, we describe the dynamics of the fission with a fully microscopic approach using the TDGCM: in a first step, the calculation of a set of static constrained meanfield states is done and the second step is the description of the dynamics by mixing the meanfield states. Due to the limitation of the calculation power, the Gaussian Overlap Approximation (GOA) has been used and the results we have obtained are in good qualitative agreement with experimental data [8]. The GOA assumes in fact two approximations: the presupposition of the overlap kernel's shape and the Taylor expansion up to the second order of the collective hamiltonian.

However, the error associated with the GOA is not known. This is why we have started two years ago the developpment of the whole formalism of the exact TDGCM.

\footnotetext{
a e-mail: marc.verriere@cea.fr

b e-mail: noel.dubray@cea.fr
}

\section{The TDGCM formalism}

The TDGCM is a beyond mean field approach that allows us to describe the collective motions of a nuclear compound system. The aim of this section is to explain the leading steps to the Hill-Wheeler equation, the main equation in the TDGCM.

\subsection{TDGCM state}

The starting point of the TDGCM is the definition of a set $\mathcal{D} \equiv\left\{\left|\varphi\left(q_{0}, q_{1}, \cdots, q_{N}\right)\right\rangle\right\}$ of states indiced by collective variables $\boldsymbol{q} \equiv\left(q_{0}, q_{1}, \cdots, q_{N-1}\right)^{\top}$.

In the case of the microscopic description of the fission process, the elements of this set can be chosen as HartreeFock-Bogoliubov (HFB) states contrained in deformation:

$$
\left\langle\varphi(\boldsymbol{q})\left|\hat{Q}_{\lambda 0}\right| \varphi(\boldsymbol{q})\right\rangle=q_{\lambda}
$$

and consequently, the set $\mathcal{D}$ is called "the deformation space" in the following. A such set is represented by a Potential Energy Surface (PES). As an example, the PES of the ${ }^{226} \mathrm{Th}$ is shown in Fig. 1. In Eq. (1), $\hat{Q}_{\lambda 0}$ names the multipolar moment of order $\lambda$ and the collective variable $q_{\lambda}$ correspond to the expected value of $\hat{Q}_{\lambda 0}$. The HFB states are calculated here in axial symmetry with an effective nucleon-nucleon interaction (Gogny D1S). $|\varphi(\boldsymbol{q})\rangle$ is developped on an optimized two-center harmonic oscillator basis $\mathcal{B}(\boldsymbol{q})$ as particle basis which depends itself on the collective variables. The time-dependent trial wave

(C) The Authors, published by EDP Sciences. This is an Open Access article distributed under the terms of the Creative Commons Attribution License 4.0 (http://creativecommons.org/licenses/by/4.0/). 


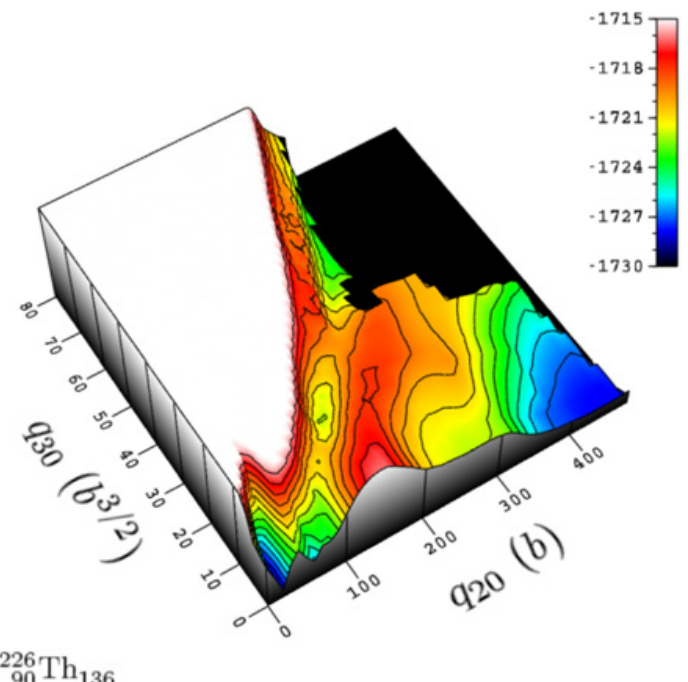

Figure 1. Potential Energy Surface of ${ }^{226} \mathrm{Th}$ constrained in elongation and asymmetry obtained by meanfield calculations (N. Dubray).

function is then defined as:

$$
|\operatorname{GCM}(t)\rangle \equiv \int_{Q} \mathrm{~d} \boldsymbol{q}|\varphi(\boldsymbol{q})\rangle f(\boldsymbol{q}, t),
$$

where $|\operatorname{GCM}(t)\rangle$ is the TDGCM state at time $t, Q$ is the set of all the collective variable values (a subset of $\mathbb{R}^{N}$ ) and $f(\boldsymbol{q}, t)$ is a complex time dependent weight function.

In order to obtain the dynamical description of the fission process, the next step is the determination of the weight function $f(\boldsymbol{q}, t)$.

\subsection{Hill-Wheeler equation}

The time dependent trial wavefunction describes a non relativistic quantum state. So, it is a solution of the Schrödinger equation:

$$
\left(\hat{H}-i \hbar \frac{\mathrm{d}}{\mathrm{d} t}\right)|\operatorname{GCM}(t)\rangle=0,
$$

with $\hat{H}$ the nuclear hamiltonian of the compound system. Applying each state $\langle\varphi(\boldsymbol{q})|$ on each side of the Eq. (3) leads to the Hill-Wheeler equation:

$$
\int_{Q} \mathrm{~d} \boldsymbol{q}^{\prime}\left(\mathcal{H}\left(\boldsymbol{q}, \boldsymbol{q}^{\prime}\right)-i \hbar \mathcal{N}\left(\boldsymbol{q}, \boldsymbol{q}^{\prime}\right) \frac{\mathrm{d}}{\mathrm{d} t}\right) f\left(\boldsymbol{q}^{\prime}, t\right)=0 .
$$

The overlap kernel $\mathcal{N}$ and the collective hamiltonian kernel $\mathcal{H}$ are defined as:

$$
\left(\begin{array}{c}
\mathcal{N}\left(\boldsymbol{q}, \boldsymbol{q}^{\prime}\right) \\
\mathcal{H}\left(\boldsymbol{q}, \boldsymbol{q}^{\prime}\right)
\end{array}\right) \equiv\left\langle\varphi(\boldsymbol{q})\left|\left(\begin{array}{c}
\mathbb{1} \\
\hat{H}
\end{array}\right)\right| \varphi\left(\boldsymbol{q}^{\prime}\right)\right\rangle .
$$

\subsection{Discretization of the deformation space}

The collective variables $\boldsymbol{q}$ and $\boldsymbol{q}^{\prime}$ in (4) are continuous and we don't have access to HFB states for every $\boldsymbol{q}$. So, the deformation space $\mathcal{D}$ is discretized. In a first step in this work, a direct discretization of the deformation space has been used: we considere now only a finite number
$M$ of constrained HFB states $\left|\varphi\left(\boldsymbol{q}_{0}\right)\right\rangle, \cdots,\left|\varphi\left(\boldsymbol{q}_{M-1}\right)\right\rangle$. The discretized Hill-Wheeler equation is:

$$
\left(\mathrm{H}-i \hbar \mathrm{N} \frac{\mathrm{d}}{\mathrm{d} t}\right) \vec{f}(t)=0
$$

with $\mathrm{N}$ the overlap and $\mathrm{H}$ the collective hamiltonian matrices, and their matrix elements are defined as:

$$
\begin{aligned}
& \mathrm{N}_{i j} \equiv \mathcal{N}\left(\boldsymbol{q}_{i}, \boldsymbol{q}_{j}\right), \\
& \mathrm{H}_{i j} \equiv \mathcal{H}\left(\boldsymbol{q}_{i}, \boldsymbol{q}_{j}\right),
\end{aligned}
$$

and $\vec{f}(t)$ is the discretized weight function:

$$
\vec{f}_{j}(t) \equiv f\left(\boldsymbol{q}_{j}, t\right)
$$

The following subsection discusses the calculation method of the overlap and the collective hamiltonian kernels.

\subsection{Collective matrices}

The first step to solve the Hill-Wheeler equation is to calculate the collective matrices $\mathrm{N}$ and $\mathrm{H}$. In this purpose, the method initially developped by Q. Haider and D. Gogny in [9] has been extended to our case of optimized two center harmonic oscillator bases in axial symmetry. This new method allows us to calculate the overlap matrix $\mathrm{N}$, the generalized density matrix $\mathrm{S}^{i j}$, and the generalized pairing matrices $\mathrm{T}^{i j}$ and $\mathrm{Y}^{i j}$ defined by equations 4.5-4.7 of [9]. With theses matrices, we can calculate every one body collective matrix thenceforth we know the expression of the matrix element of the associated operator in the harmonic oscillator basis in $\boldsymbol{q}_{i}$ or $\boldsymbol{q}_{j}$.

\subsection{Dynamical description}

To solve the Hill-Wheeler Eq. (6) with a small number of meanfield states, there is no need of a time scheme: the Hill-Wheeler equation is a linear differential equation of order one. However, the HFB states in the deformation space are not independent. After a proper orthonormalization of the HFB states using a diaonalization of the overlap matrix $\mathrm{N}$ and the deletion of the eigenstates of $\mathrm{N}$ associated with a small eigenvalue $\lambda$ $\left(\lambda<\epsilon\right.$ with $\epsilon \sim 10^{-4}$ ), the Hill-Wheeler equation can be written on the form:

$$
\left(\tilde{\mathrm{H}}-i \hbar \frac{\mathrm{d}}{\mathrm{d} t}\right) \vec{g}(t)=0 .
$$

$\tilde{\mathrm{H}}$ is the expression of the collective hamiltonian in the orthonormal basis which diagonalizes $\mathrm{N}$ and $\vec{g}(t)$ is the time dependent weight vector in this basis and contains all the information about the TDGCM state $|\operatorname{GCM}(t)\rangle$. The expression of $\vec{g}(t)$ can be found analytically in this case:

$$
\vec{g}(t)=\exp \left(-\frac{i t}{\hbar} \tilde{\mathrm{H}}\right) \vec{g}(t=0) .
$$

\section{Results for the reaction ${ }^{235} U(n, f)$}

The results presented here are obtained with a set of HFB states constrained in elongation for the reaction ${ }^{235} \mathrm{U}(\mathrm{n}, \mathrm{f})$. 


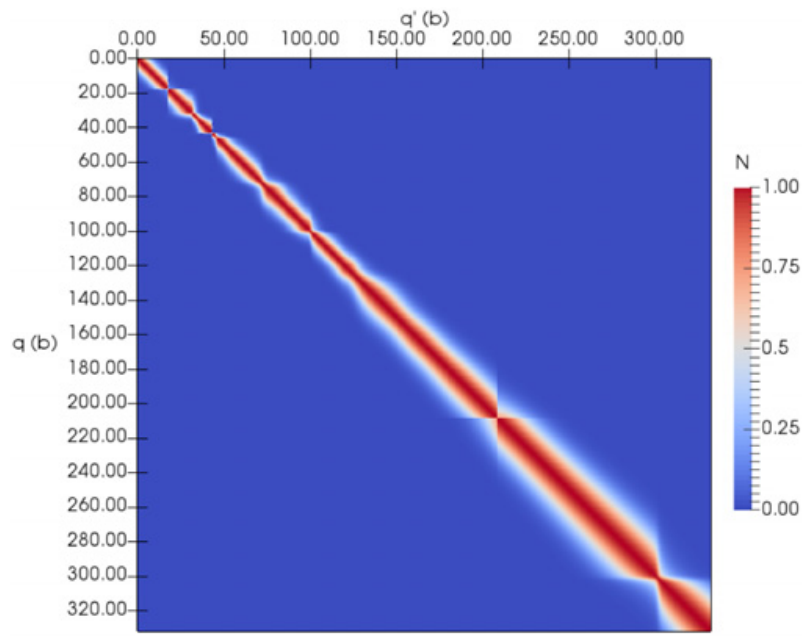

Figure 2. Overlaps calculated for each pair $\left(\left|\varphi\left(\boldsymbol{q}=q_{20}\right)\right\rangle,\left|\varphi\left(\boldsymbol{q}^{\prime}=q_{20}^{\prime}\right)\right\rangle\right)$ of HFB states constrained in elongation.

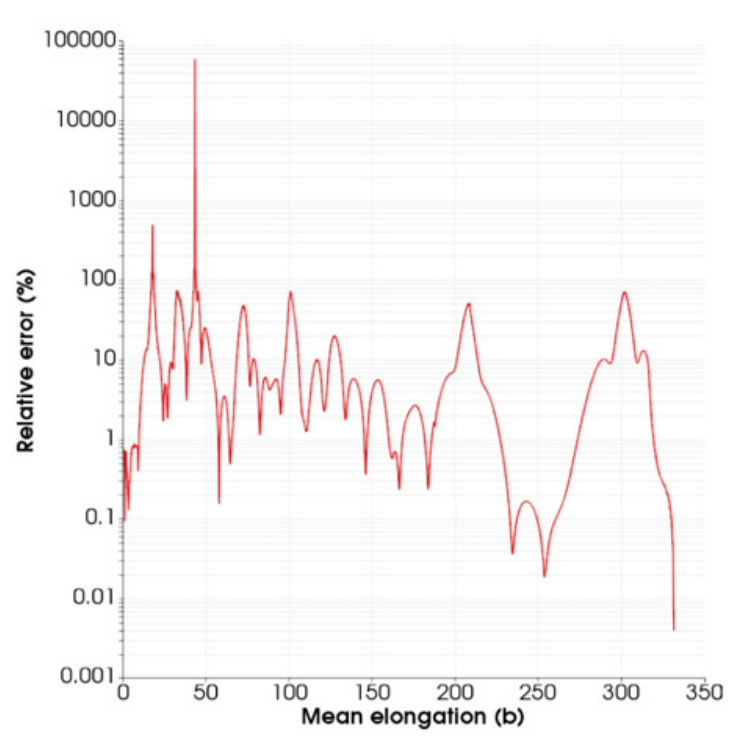

Figure 3. Relative error between the exact and the TDGCM+GOA overlaps.

\subsection{Collective matrices}

The overlap matrix is shown in Fig. 2. Its values decrease rapidely according to $\boldsymbol{q}-\boldsymbol{q}^{\prime}$, where $\boldsymbol{q} \equiv q_{20}$ and $\boldsymbol{q}^{\prime} \equiv q_{20}^{\prime}$. There are singularities around $\overline{\boldsymbol{q}}=\overline{q_{20}} \in\left\{25 \mathrm{fm}^{2}, 50 \mathrm{fm}^{2}, 100 \mathrm{fm}^{2}, 210 \mathrm{fm}^{2}, 310 \mathrm{fm}^{2}\right\}$ with $\overline{\boldsymbol{q}} \equiv \frac{1}{2}\left(\boldsymbol{q}+\boldsymbol{q}^{\prime}\right)$, due to fast variation or discontinuities $[10]$ in the deformation space.

We have calculated the relative error between the exact overlap and the TDGCM+GOA ones (without metric correction) by a gaussian fit on the exact overlap. The relative errors $\varepsilon(\overline{\boldsymbol{q}})$ for each $\overline{\boldsymbol{q}}$ is defined as:

$$
\varepsilon(\overline{\boldsymbol{q}}) \equiv \frac{\| \mathrm{N}_{\bar{q}}^{\mathrm{calc}}-\mathrm{N}_{\bar{q}}^{\mathrm{fit} \|}}{\left\|\mathrm{N}_{\bar{q}}^{\mathrm{fit}}\right\|} .
$$

The results we have obtained are represented on the Fig. 3. Around singularities into the exact overlap matrix, we can see a relative error greater than $50 \%$ and up to 3 order of magnitudes.

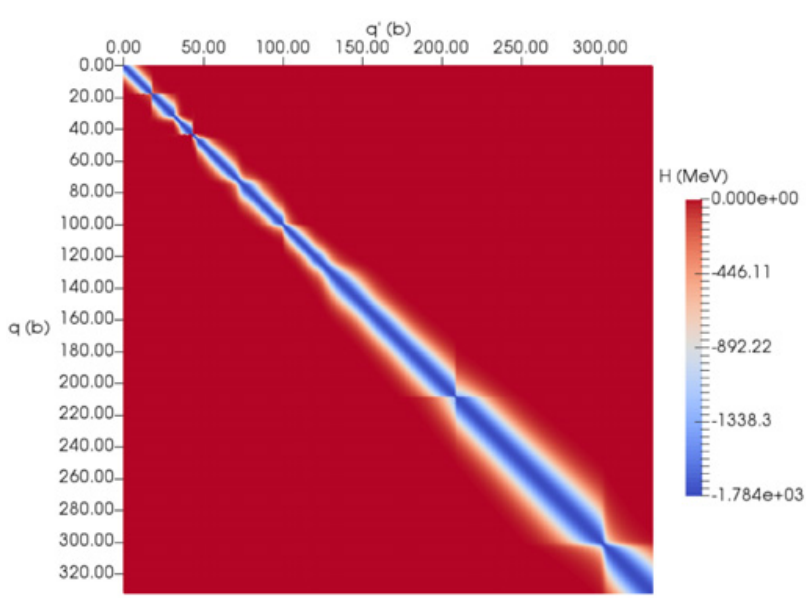

Figure 4. Collective hamiltonian values calculated for each pair $\left(\left|\varphi\left(\boldsymbol{q}=q_{20}\right)\right\rangle,\left|\varphi\left(\boldsymbol{q}^{\prime}=q_{20}^{\prime}\right)\right\rangle\right)$ of HFB states constrained in elongation.

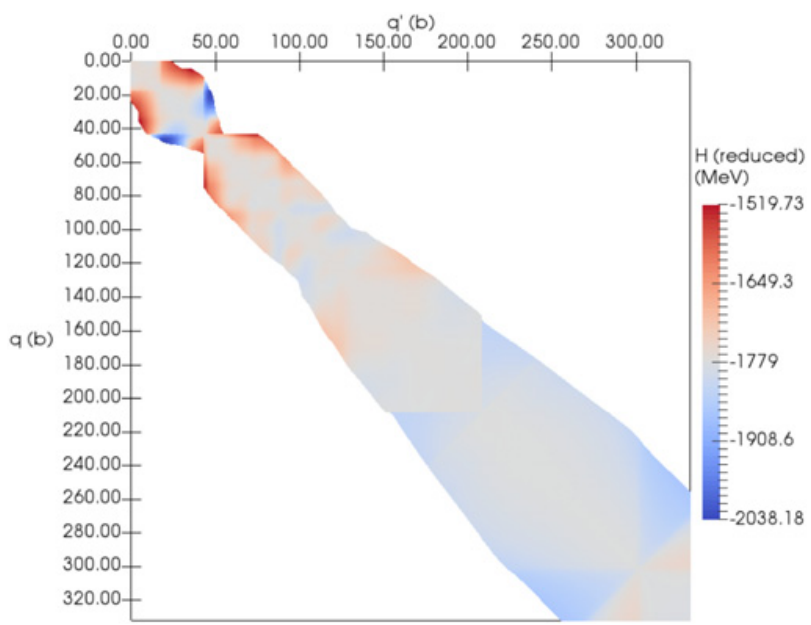

Figure 5. Reduced collective hamiltonian values, where the white areas correspond to the pair of HFB states with an overlap lower than $10^{-8}$.

The collective hamiltonian matrix is shown in Fig. 4. It is calculated for every pair of HFB state for which the overlap is greater than $10^{-8}$, and the other values of the collective hamiltonian are set to 0 . The reduced collective hamiltonian $\mathrm{H}^{\text {red }}$, defined as:

$$
\mathrm{H}_{i j}^{\mathrm{red}} \equiv \frac{\mathrm{H}_{i j}}{\mathrm{~N}_{i j}}
$$

allows us to see closely the variations into the hamiltonian and it is shown in Fig. 5.

\subsection{Dynamical description}

The vector $\vec{g}(t=0)$ is chosen to have the two properties:

1. $(\mathrm{N} \vec{f}(t=0))_{i}$, the initial probability to be in the HFB state in $\boldsymbol{q}_{i}$, is a gaussian centered in the first well of potential;

2. the energy of the TDGCM state is slightly above the fission barrier. 
We have observed the influence of fast variations of the distance $\mathrm{d}\left(\boldsymbol{q}, \boldsymbol{q}^{\prime}\right)$ associated with the overlaps, defined by:

$$
\mathrm{d}\left(\boldsymbol{q}, \boldsymbol{q}^{\prime}\right) \equiv \sqrt{2-2\left\langle\varphi(\boldsymbol{q}) \mid \varphi\left(\boldsymbol{q}^{\prime}\right)\right\rangle}
$$

between neighbor HFB states in the deformation space.

\section{Conclusions and remarks}

This work is a first step to the description of the fission process using the TDGCM. The calculation of observables (fission yields, neutron emission multiplicities, ...) remains an open question in this case, but several possibilities are explored. The next steps of this work are the calculation of these observables using sets of HFB states constrained in elongation and mass asymmetry, and the implementation of a new solver using a time scheme (Crank-Nicolson for example).

There are also possible continuations, as the addition of the intrinsic excitations to break the adiabatic hypothesis in our model.

\section{References}

[1] N. Bohr, J.A. Wheeler, Phys. Rev. 56, 426 (1939)

[2] P. Möller, A.J. Sierk, T. Ichikawa, A. Iwamoto, R. Bengtsson, H. Uhrenholt, S. Åberg, Phys. Rev. C 79, 064304 (2009)

[3] P.A.M. Dirac, Mathematical Proceedings of the Cambridge Philosophical Society 26, 376 (1930)

[4] J.W. Negele, S.E. Koonin, P. Möller, J.R. Nix, A.J. Sierk, Phys. Rev. C 17, 1098 (1978)

[5] P. Goddard, P. Stevenson, A. Rios, Phys. Rev. C 92, $054610(2015)$

[6] J.J. Griffin, J.A. Wheeler, Phys. Rev. 108, 311 (1957)

[7] P.G. Reinhard, K. Goeke, Reports on Progress in Physics 50, 1 (1987)

[8] D. Regnier, N. Dubray, N. Schunck, M. Verrière, Phys. Rev. C 93, 054611 (2016)

[9] Q. Haider, D. Gogny, Journal of Physics G: Nuclear and Particle Physics 18, 993 (1992)

[10] N. Dubray, D. Regnier, Computer Physics Communications 183, 2035 (2012) 